\title{
Determination of Chamber and Piston Geometry for a Rotary Type Expansion Machine
}

\author{
Andrey Perminov \\ Department of Electric Drive and Auto- \\ mation Systems \\ Pskov State University \\ Pskov, Russia \\ alp-mail@mail.ru \\ Sergey Tikhonov \\ Department of Mechanics and Motor \\ Trantsport Service \\ Pskov State University \\ Pskov, Russia \\ sit42@rambler.ru
}

\author{
Yury Zhuravlev \\ Department of Road Construction \\ Pskov State University \\ Pskov, Russia \\ drakon426@mail.ru
}

Alexander Ilyin

Department of Electric Drive and Automation Systems

Pskov State University

Pskov, Russia

al.ilyin@yandex.ru

\author{
Yury Lukyanov \\ Department of Electric Drive and Auto- \\ mation Systems \\ Pskov State University \\ Pskov, Russia \\ luk-yra@mail.ru
}

\begin{abstract}
The article deals with the determination of chamber and piston geometry for a rotary expansion machine. Analytical expressions for the working surface area of piston and for chamber volume are derived. Three defining parameters of the expansion machine were obtained: gas dynamic machine constant $\mathrm{Cp}$; constant M0 connecting torque and $\mathrm{Cp}$; the main dimensionless parameter of the machine $\mathrm{Q} 0$, which relates the volume of the chamber and the torque.
\end{abstract}

Keywords-power generation system, rotary engine, lever-cam motion converter.

\section{INTRODUCTION}

The subject of this article is the rotary type expansion machine protected by patent RU2619391 [1].

Fig. 1 shows a piston $1(\mathrm{AB})$, which is in a fixed position given by the polar angle $\alpha=-45^{\circ}$ between the interpolar radius $O A=\rho_{A}=\rho(\alpha)$ and the axis $O x$. In this position, the volume of the chamber is maximum, while

$$
\rho_{A}=\rho_{B}=L \cdot \sin (a+b \cdot \cos 2 \alpha)=\frac{\sqrt{2}}{2} L,
$$

where $L$ is the distance between the axes of the piston hinges, $a=\pi / 4 ; b=\pi / 4-\psi_{\min } / 2$, and where $\psi_{\min }$ is the minimum angle between the pistons.

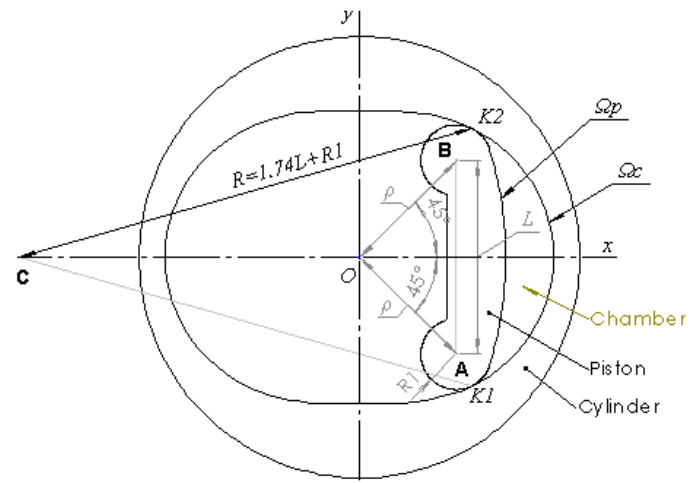

Fig. 1. Piston in a fixed position given by the polar angle
The piston profile $\Omega_{P}$ relates to the equidistant surface of the cylinder $\Omega_{C}$ at two points $K_{1}$ and $K_{2}$. A chamber is formed between these points and the surfaces, the volume of which $V_{K}$ with $\alpha=-45^{\circ}$ should have the specified maximum value $V_{0}$, i.e.

$$
V_{K}=V_{K}\left(L, R_{l}, t\right)=t \cdot S_{K}\left(L, R_{l}\right)=V_{0},
$$

where $t$ is the piston thickness; $R_{1}$ is the radius of the end curves of the piston; $S_{K}\left(L, R_{p}\right)$ is the chamber area.

The task is to obtain the specified value of the chamber volume with different variants of the values of three geometric parameters $L, R_{1}, t$.

To do this, we need to compile some functional and get values of parameters $L, R_{l}, t$, which deliver the extremum to the functional.

\section{ANALYSIS OF GEOMETRIC CONSTRAINTS}

\section{A. Preliminary notes}

For the presence of extremum the influence of the three quantities $L, R_{l}, t$, must be multidirectional.

We will try to solve the problem of determining the geometric dimensions of $L, R_{l}, t$, based on the analysis of the restrictions imposed on the geometry of the machine for one reason or another. Consider three of them: 1) cross section size of cylinder; 2) volume of chamber; 3 ) torque.

\section{B. Cross section size of cylinder}

When making a cylinder from a single piece, the diameter must be available for processing equipment. From this point of view, and also proceeding from economy of metal and reduction in price, the size of the cylinder $L_{C}$ (Fig. 2) should be as small as possible. Let the upper value of the size of the cylinder $L_{C}$ be given, which we denote by $L_{C}$. 


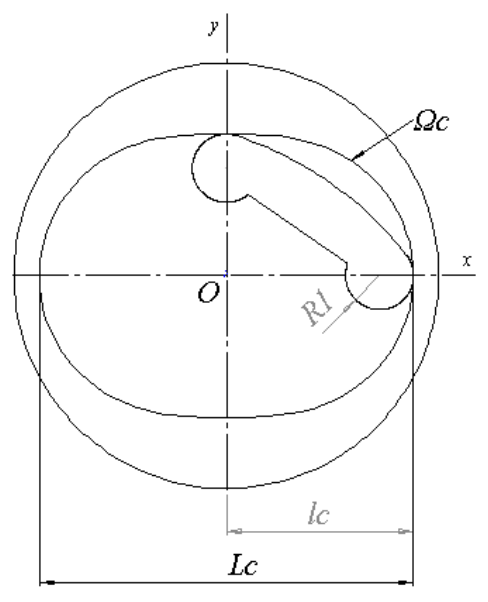

Fig. 2. Piston in a fixed position given by the polar angle $\alpha=0^{\circ}$

From Fig. 2 follows

$$
\begin{gathered}
\frac{1}{2} L_{c}=\rho_{A}(0)+R_{1}= \\
L \sin (a+b)+R_{1}=0.819 L+R_{1} .
\end{gathered}
$$
ity

The constraint in question is described by the inequal-

$$
0.819 L+R_{1} \leq \hat{L}_{c} / 2
$$

\section{Volume of chamber}

The chamber volume is a pi-periodic function of the angle of rotation of the shaft $\alpha$, which varies in the range $V_{\min } \ldots V_{\max }$. The term "chamber volume" is understood as the value $V_{\max }$, which is described by the approximate expression [2]

$V_{K}\left(L, R_{l}, t\right)=\left(0.15 L^{2}+0.54 L R_{1}+0.49 R_{1}^{2}\right)$

If based on thermal calculations, this volume must be equal to the specified value $V_{0}$, we will have a limitation

$$
V_{\max }\left(L, R_{1}, t\right)=V_{0} \text {. }
$$

\section{Torque}

The torque $M_{T}$ is a $\pi 2$-periodic function of the angle of rotation of the shaft, which, being expanded in a Fourier series, has a constant component

$$
a_{0}=\frac{1}{\pi / 2} \int_{0}^{\pi / 2} M_{T}(\alpha) d \alpha .
$$

For a given nominal power $N_{N}$ and nominal angular velocity $\omega_{N}$, the value can be considered known and equal to [3]

$$
a_{0}=\frac{N_{N}}{\omega_{N}}
$$

According to [3] for torque

$$
\begin{aligned}
& M_{T}(\alpha)=\frac{L}{2}\left[\left(F_{2}+F_{4}\right)(1-2 b \sin 2 \alpha)-\right. \\
& \left.\left(F_{1}+F_{3}\right)(1+2 b \sin 2 \alpha)\right] \sin (2 b \cos 2 \alpha),
\end{aligned}
$$

where $2 b=2 \cdot 0.1749=0.35$. Considering that the gas forces acting on the pistons are $F_{3}=F_{1}=p_{1}(\alpha) S_{P} F_{4}=F_{2}=p_{2}$ $(\alpha) S_{p}$ where $p_{1}(\alpha)$ and $p_{2}(\alpha)$ are the gas pressure on the pistons 1 and 3, and 2 and 4 , respectively, and where $S_{P}$ is the working surface area of the piston $S_{P}=2 \mid y_{K 1}$ $\mid t=2\left(0.5 L+0.9 R_{1}\right) t$, or $S_{P}=\left(L+1.8 R_{1}\right) t$. Expression [9] takes the form

$$
\begin{aligned}
& M_{T}(\alpha)=\left(L^{2}+1,8 L R_{1}\right) t\left[p_{2}(\alpha)(1-0.35 \sin 2 \alpha)-\right. \\
& \left.p_{1}(\alpha)(1+0.35 \sin 2 \alpha)\right] \quad \sin (0.35 \cos 2 \alpha) .
\end{aligned}
$$

Substituting (10) into (7) and introducing the designation of a parameter

$$
\begin{aligned}
& C P=\frac{2}{\pi} \int_{0}^{\pi / 2} p 2(\alpha)(1-0.35 \sin 2 \alpha)-p 1(\alpha) \\
& (1+0.35 \sin 2 \alpha)] \sin (0.35 \cos 2 \alpha) d \alpha
\end{aligned}
$$

We arrive at a torque-induced geometry limitation.

$$
\left(L^{2}+1,8 L R_{1}\right) t=\frac{a_{0}}{C_{p}}
$$

Note that the constant $C_{P}$ is an important gas-dynamic parameter of machine. It depend only on the gas pressure in the chambers and depend on the geometry of the chambers.

\section{DETERMINATION OF BASIC GEOMETRIC DIMENSIONS}

The main dimensions of the machine are three parameters: the inter-joint distance on the piston $A B=L$, the radius of the end curves of piston $R_{l}$ and the thickness of piston $t$.

\section{A. Initial equations}

Three equations (5), (12) and (6), respectively, describing the restrictions on the size of the cylinder $L_{c}$, on the torque $M_{T}$ and on the volume of the chamber $V_{0}$ :

$$
\begin{aligned}
& 0.819 L+R_{1} \leq \hat{l}_{c}, \\
& \left(L^{2}+1,8 L R_{1}\right) t=m_{\curvearrowleft}, \\
& \left(0.15 L^{2}+0.54 L R_{1}+0.49 R_{1}^{2}\right) t=V_{0} .
\end{aligned}
$$

Here, for the sake of brevity, new notation has been introduced $m_{0}=a_{0} / C_{P}, l_{C}^{\wedge}=L \hat{C}^{\wedge}$, in which $a_{0}$ and $C_{p}$ are defined by (8) and (11), respectively.

If we specify the size of the cylinder $l \hat{C}_{C}=l$, the inequality in (13) becomes equal to $0.819 L+R_{l}=l$.

The formulation of the solution of the problem on the basis of three equations, two of which are non-linear, may be different. There is no guarantee that a solution exists. 
It is quite obvious, for example, that with a very small cylinder size $l_{c} \hat{C}$, it is not possible to create the required torque $a_{0}$, or the specified volume of the chamber $V_{0}$.

\section{B. Common Solution}

Let cylinder size $l_{C}^{\wedge}=l$ be given. Then we have a system of three algebraic equations.

$$
\begin{aligned}
& 0.819 L+R_{1}=l, \\
& \left(\mathrm{~L}^{2}+1.8 \mathrm{LR}_{1}\right) \mathrm{t}=\mathrm{m}_{0}, \\
& \left(0.15 \mathrm{~L}^{2}+0.54 \mathrm{~L} \mathrm{R}_{1}+0.49 \mathrm{R}_{1}{ }^{2}\right) \mathrm{t}=\mathrm{V}_{0}
\end{aligned}
$$

From the first equation we express $R_{1}$ through $L$ : $R_{1}=l-0.819 L$. From the second equation we express $t$ through $L$ :

$t=\frac{m_{0}}{L^{3}+1.8 L(l-0.819 L)}$, or $t=\frac{m_{0}}{1.8 L l-0.474 L^{2}}$

, or Substituting into the third equation:

$\left[0.15 L^{2}+0.54 L(I-0.819 L)+0.49(I-0.819 L)^{2}\right] \frac{m_{0}}{1.8 L l-0.474 L^{2}}=V_{0}$

By opening the brackets and grouping the terms, we get the equality

$0.0367 L^{2}-0.263 L l+0.49 l^{2}=V_{0} / m_{0}\left(1.8 L l-0.474 L^{2}\right), \quad$ from which the quadratic equation follows on the size of $\mathrm{L}$ :

$$
a L^{2}+b L+c=0
$$

where $=0.474 V_{d} / m_{0}+0.0367, \mathrm{~b}=-\left(1.8 \mathrm{~V}_{0} / \mathrm{m}_{0}+0.263\right) 1$, $\mathrm{c}=0.49 \mathrm{l}^{2}$, whose solution has a well-known appearance

$$
L_{1,2}=\frac{-b \pm \sqrt{b^{2}-4 a c}}{2 a}
$$

Considering that $V_{0}$ is the volume of the camera and $\mathrm{m}_{0}=\mathrm{a}_{0} \mathrm{C}_{\mathrm{p}}$ is a parameter characterizing the torque according to (10) and having the dimension $\mathrm{m}^{3}$ we introduce the dimensionless machine parameter

$$
q_{0}=\frac{V_{0}}{m_{0}}
$$

We write the coefficients of equation (15) in the form

$$
\begin{gathered}
a=0.474 q_{0}+0.0367, \\
b=-\left(1.8 q_{0}+0.263\right) l, \\
c=0.49 l^{2} .
\end{gathered}
$$

\section{Common Solution Analysis}

It is known that the number of real solutions of equation (15) depends on the discriminant sign $D=b^{2}$ $-4 a c$ : if $D>0$, then there are 2 solutions (2 real roots); $D=0$, then there is 1 solution ( 2 matching roots); $D<0$, then there are 0 solutions ( 2 imaginary roots).

We make the discriminant the coefficients of which are determined by (17):

$$
D=l^{2}\left(3.24 q_{0}{ }^{2}+0.016 q_{0}-0.0027\right)
$$

The value of the parameter $q_{0}=V / m_{0}$, for which $D=0$ . Rejecting the negative root, we get

$$
q_{0}=\frac{V_{0}}{m_{0}}=0.0265
$$

When $\mathrm{q}_{0}=0.0265$ there is one solution, i.e. two coincident positive roots $L_{1}=L_{2}=-b / 2 a=3.151$ When $q_{0}<0.0265$ there are no solutions; at $q_{0}>0.0265$ there is one positive solution for $L$. At the same time, the presence of a solution for $L$ does not guarantee the presence of positive solutions for $R_{1}$ and $t$.

For convenience, let's move on to the dimensionless variables.

$$
L^{\prime}=\frac{L}{l}, R_{1}^{\prime}=\frac{R_{1}}{l}, t^{\prime}=\frac{t}{l} .
$$

values will be positive if the inequality

$l\left(1-0.819 L^{\prime}\right)>0$ is satisfied, or the condition $L^{\prime}<1.22$.

Find the value of $\mathrm{q}_{0}$, for which, $L^{\prime}<1.22, R^{\prime}=0$.

After the transformations we get the solution $q_{0}=0.15$.

Thus, for $\mathrm{R}_{1}$ to be greater than zero, the machine parameter connecting the chamber volume and torque $\mathrm{q}_{0}=\mathrm{V}_{0} / \mathrm{m}_{0}=\mathrm{V}_{0} /\left(\mathrm{a}_{0} / \mathrm{C}_{\mathrm{p}}\right)$ must be greater than 0.15 .

The results of calculations of dependences $\mathrm{L}^{\prime}=\mathrm{L} / \mathrm{l}$, $\mathrm{R} \_1^{\prime}=\mathrm{R} \_1 / 1$ and $\mathrm{t}^{\prime}=\mathrm{t} / \mathrm{l}$ multiplied by $\mathrm{l}^{3} / \mathrm{m}^{0}$, from $\mathrm{q}_{0}$ are shown in Table 1

TABLE 1

\begin{tabular}{|c|c|c|c|c|}
\hline $\mathrm{q}_{0}$ & $\mathrm{~L}^{\prime}=\mathrm{L} / \mathrm{l}$ & $\mathrm{R}_{1}^{\prime}=\mathrm{R}_{1} / \mathrm{l}$ & $\mathrm{R}_{1} / \mathrm{L}$ & $\left(\mathrm{t}^{\prime} \mathrm{l}^{3}\right) / \mathrm{m}_{0}$ \\
\hline 0.15 & 1.22 & 0 & 0 & 0.45 \\
\hline 0.175 & 1.00 & 0.181 & 0.181 & 0.754 \\
\hline 0.20 & 0.996 & 0.184 & 0.185 & 0.756 \\
\hline 0.25 & 0.841 & 0.311 & 0.370 & 0.850 \\
\hline 0.30 & 0.730 & 0.402 & 0.550 & 0.940 \\
\hline 0.50 & 0.475 & 0.610 & 1.280 & 1.340 \\
\hline 1.00 & 0.254 & 0.792 & 3.120 & 2.340 \\
\hline
\end{tabular}

The table has a range of $0.175 \leq q_{0} \leq 0.3$. This range has practical interest due to the fact that the ratio $R_{1} / L$ from design considerations should not be greater than 0.5 . Graphically, this range is shown in Fig. 3. The dotted line shows the relation $R_{l} / L$. 


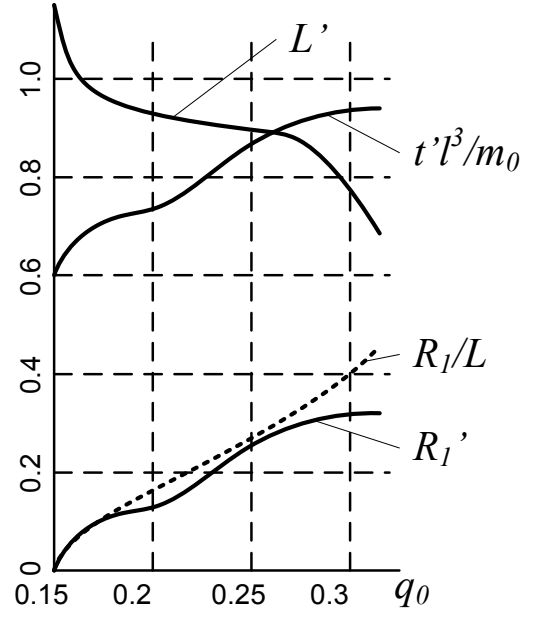

Fig. 3.

\section{Conclusions}

The main result is the identification of several important parameters of rotary expansion machine.

First of all, this is the gas-dynamic constant $C_{P}\left(H / \mathrm{M}^{2}\right)$ defined by (11). It depends only on the initial and final pressure of the gas and does not depend on the geometrical dimensions of chamber and piston.

The second parameter is $m_{0}=a_{0} / C_{P}\left(m^{3}\right)$, which relates the torque and the gas dynamic constant.

The third, which can be called the main dimensionless rotary expansion machine parameter, is $q_{0}=V / m_{0}$, which relates the chamber volume and torque, and, as was established above, its value should be in the range of $0.15<\mathrm{q}_{0}<0.3$.

\section{REFERENCES}

[1] RF Patent 2619391 for an invention. Int. Cl. F01C1/44. Rotary Expantion Machine / Y.N. Lukyanov, Y.N. Zhuravlev et al. Publ. 15.05.2017.

[2] Y.Zhuravlev, A.Perminov, Y.Lukyanov, S.Tikhonov, A.Ilyin, S.Semyonov, Optimization of Mechanical Strength of Rotary-Vane Engine. "Environment. Technology. Resources - Proceedings of the 11th International Scientific and Practical Conference", 2017. DOI: 10.17770 .

[3] S.Semyonov, S.Tikhonov, Y.Lukyanov, A.Perminov, Analysis Method of Calculation Parameters of Heat and Mass Transfer Processes in the Stirling Engine. "Environment. Technology. Resources - Proceedings of the 11th International Scientific and Practical Conference", 2017.

[4] Y. N. Zhuravlev, I. V. Plokhov, Y. N. Lukyanov, and others, Calculation and Design of Rotaty-blade Machine with External Heat Supply. Monogrphy. Pskov, 2012.

[5] Y. N. Zhuravlev, I. V. Plokhov, Y. N. Lukyanov, and others, Mathematical Models of Physical Processes in Rotary-blade Engine with External Heat Supply. Monogrphy. Pskov, 2012.

[6] Y. N. Zhuravlev, A. L. Perminov, Y. N. Lukyanov, S. I. Tikhonov, S. N. Semenov, Minimization of Mechanical Strength of Rotary-blade Heat Engine with Cam-and-lever Motion Transformer. Bulletin of Pskov State University. Series: Engineering scinces, 2016. \# 4.

[7] RF Patent 2374526 for an invention. Int. Cl. F16H25/04. Mechanism for converting motion / Y.N. Lukyanov, Y.N. Zhuravlev et al. Publ. 27.11.2009. Bull. Number 33.

[8] RF Patent 2387844 for an invention. Int. Cl. F01G1/077, F02G1/044. Rotary-vane engine with an external supply of heat / .N. Lukyanov, Y.N. Zhuravlev et al. Publ. 27.04.2010. Bull. Number 12 .

[9] Perminov A., Lukyanov Y., Tikhonov S., Ilyin A. Thermodynamic cycle with two-component working fluid. "Environment. Technology. Resources - Proceedings of the 11th International Scientific and Practical Conference", 2015. DOI: 10.17770/etr2015 vol2.261.

[10] A. L. Perminov, A. A. Khitrov, A. I. Khitrov, Mechatronic System "Magneto-electric Synchro Motor - Active Rectifier" for Autonomous Energy Station Using Rotary-blade Machine with External Heat Supply. "Proceedings of VII International (VIII Russian) Conference on Automotization Electrical Drives AEP-2012", Ivanovo State Energy University, Ivanovo, 2012. P. 330-335

[11] A. L. Perminov, Y. N. Lukyanov, S. I. Tikhonov, Electrogenerators fot Energy Sets. "Electrical Engineering. Electrical Energy. Information Techlogies. Proceedins of I International Research and Prctice Seminar", Pskov. 2018. P. 52-55. 